\title{
Redefining Esthetics: The Implant Way
}

\author{
Prabal Sharma' Bhumika Sharma' Sumit Makkar² \\ ${ }^{1}$ Department of Prosthodontics Including Crown and Bridge, MM \\ College of Dental Sciences and Research, Ambala, Haryana, India \\ ${ }^{2}$ Private Practitioner, Noida, Uttar Pradesh, India
}

\author{
Sandeep Kalra ${ }^{1}$
}

Dent J Adv Stud 2021;9:105-109.

\begin{abstract}
Address for correspondence Prabal Sharma, MDS, 1491-B, Modern Colony, Near I.T.I, Yamunanagar, Haryana 135001, India (e-mail: prabalsharma.3@gmail.com).
\end{abstract}

\begin{abstract}
Keywords

- anterior maxilla

- aesthetics

- restoration

- harmonious

- concavity

Alteration in conventional techniques in anterior maxilla is essential for making a restoration look more natural and harmonious with the rest of the mouth, as this area is more prone to aesthetic failure. The visibility of anterior crowns is obviously more, making it necessary for a restoration to blend with the natural dentition. This makes a person's smile more pleasant and aesthetic. Proper risk assessment must be considered while placing implants in anterior maxillary region as any deficiency in bone and soft tissue could compromise the aesthetics as well as overall success of a dental implant restoration. Timing of implant placement also plays a crucial role in providing aesthetic success in this region. This article presents a case where implant placement is done, enhancing the aesthetics as well as function in the anterior maxillary region.
\end{abstract}

\section{Introduction}

Dentistry has witnessed a significant evolution with the development of newer restorative materials and advanced techniques which have led to an unprecedented improvement in aesthetic rehabilitation. Using a comprehensive approach in diagnosing and treatment planning of the partial edentulous cases can help to achieve the aesthetics that best enhance the overall facial appearance of the patient with additional benefit of enhanced oral health. ${ }^{1}$ Complications related to occlusion, hard and soft tissues, and muscular and articular components are often associated with partial edentulism. ${ }^{2}$ Although, maintaining aesthetics in rehabilitation of anterior edentulous patients remains a challenge due to the complexities of maintaining an adequate framework of hard and soft tissue architecture, which is ultimately required for the restorative phase of treatment. One of the major difficulties in implant-prosthetic therapy is obtaining an aesthetically pleasing result as compared with tooth-supported restorations As the implant-supported restoration has to eventually harmonize the individual's frame of the smile and face. ${ }^{3}$
Proper treatment planning is required during implant therapy in the anterior maxilla due to unique conditions present in this zone. While the overall success of an implant therapy majorly depends on the hard and soft tissue dimensions available, implant positioning with respect to apicocoronal, mesiodistal, and buccolingual positions with correct angulations of implants. ${ }^{4}$ Maxillary anteriors are more prone to root fractures, recurrent failures of root canals, dental caries, and periodontal disease; therefore, the treatment plan for maxillary anterior teeth is more strenuous, leaving this region compromised in bone and soft tissue support. ${ }^{5}$

This case report describes the replacement of missing maxillary anterior teeth with the implant-ceramic restorations maintaining the aesthetic as well as functional harmony.

\section{Case Report}

A 29-year-old man presented to the Department of Prosthodontics with the need of aesthetic crowns in the anterior region of upper arch ( - Fig. 1). Conducting a
DOI https://doi.org/ $10.1055 / \mathrm{s}-0041-1731103$ ISSN 2321-1482
(C) 2021. Bhojia Dental College and Hospital affiliated to Himachal Pradesh University.

This is an open access article published by Thieme under the terms of the Creative Commons Attribution-NonDerivative-NonCommercial-License, permitting copying and reproduction so long as the original work is given appropriate credit. Contents may not be used for commercial purposes, or adapted, remixed, transformed or built upon. (https://creativecommons.org/licenses/by-nc-nd/4.0/).

Thieme Medical and Scientific Publishers Pvt. Ltd. A-12, 2nd Floor, Sector 2, Noida-201301 UP, India 


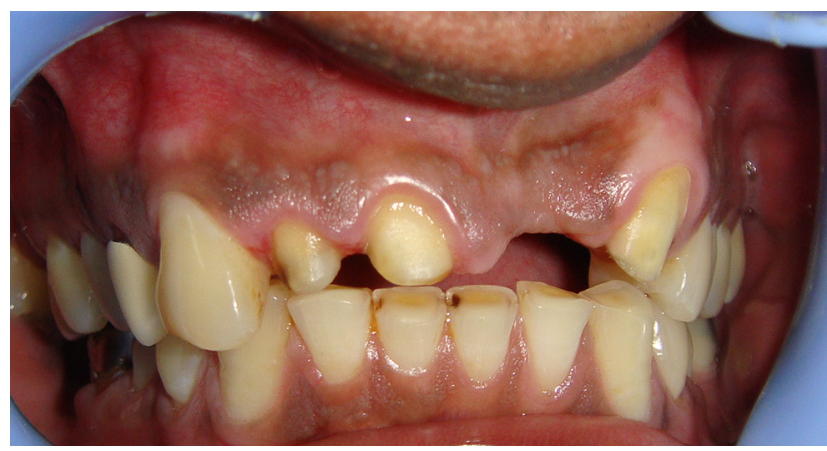

Fig. 1 Intraoral view of the edentulous area.

thorough medical history it was found that the patient was moderately built and nourished, with no signs of any systemic illness.

\section{Dental History}

A detailed dental history was taken, which revealed that two teeth (i.e., 21 and 22) were lost due to a trauma 5 years ago and the adjacent three teeth were prepared at that time due to need for restorative intervention. Porcelain fused to metal fixed partial denture was then given on these prepared and missing tooth.

\section{Clinical Examination}

Clinical examination was conducted which reported the following:

Extraoral: Absence of any swelling with symmetrical facial profile. Temporomandibular joint (TMJ) was also found to be normal without any clicking or deviation while mouth opening.

Intraoral: The teeth 21 and 22 were missing with adequate quantity and quality of alveolar bone present in this edentulous area. Concavity in the labial ridge was interpreted as labial dehiscence.

Periodontal status: Periodontal status was good as there was no sign of inflammation, periodontal pockets, or tooth mobility with a maintained oral hygiene.

Occlusion: Occlusion was stable with normal overbite and overjet.

Radiographic evaluation: Remaining teeth were in a healthy state. Adequate bone height of $13 \mathrm{~mm}$ was present. Bony defect was detected in 21 and 22 tooth region needing a definite bone augmentation to attain long-term success.

Preoperative procedures consisting of radiographic analysis, diagnostic casts, oral prophylaxis, and routine blood investigations were performed, followed by a brief case history.

\section{Treatment Options}

The treatment options were discussed with the patient. Moreover, treatment cost and time with pros and cons of each treatment option were thoroughly explained to the patient. The treatment options were summarized as follows:

- Fixed partial denture (either porcelain fused to metal crowns or all ceramic crowns).
- Implant/implants in an edentulous area (i.e., 21 and 22 and fixed prosthesis on prepared teeth; either porcelain fused to metal crowns or all ceramic crowns).

- Extraction of the prepared teeth followed with the placement of implants for the respective missing teeth (porcelain fused to metal crowns or all ceramic crowns).

The patient decided to pursue with the implant placement for the missing teeth with all ceramic bridge as the patient wanted fixed prosthesis with no metal visibility. Duly written consent was obtained from the patient followed by the treatment plan discussion.

\section{Treatment Planning}

Porcelain fused to metal (PFM) bridge was removed using a crown remover. Maxillary and mandibular diagnostic impressions were made; casts were obtained and wax-up was done to provide an adequate restorative treatment plan. A proper surgical plan was made with the help of cone beam computed tomography (CBCT) scan and therefore a radiographic/surgical guide was also fabricated for proper implant fixture placement.

CBCT examination revealed a good height of available bone $(13 \mathrm{~mm})$ whereas insufficient width of the ridge in the area where implant has to be placed. Hence it was decided to first expand the bone using bone expanders followed by placement of an implant with the dimension of $3.3 \mathrm{~mm} \times 10 \mathrm{~mm}$.

\section{Implant Placement}

A sharp midcrestal incision was given and a full-thickness flap was raised to expose the ridge crest, which was $\sim 4 \mathrm{~mm}$ buccopalatally (-Fig. 2). The bone expanders (bone compression kit, MIS Implant, Israel) were used for bone expansion. The expanders were inserted in intervals, pausing for 10 seconds to allow time for the bone to expand ( $\mathbf{- F i g . 3}$ ). These expanders are self-tapered, with diameters of 2.6, 3.0, 3.4, 3.8, 4.3, 1.6 , and $2.6 \mathrm{~mm}$ in the apex. Finally the implant fixture of dimensions $3.3 \mathrm{~mm} \times 10 \mathrm{~mm}$ was placed (Alpha Biocare SP, Israel) with the primary stability $>35 \mathrm{Ncm}$, followed by the placement of the cover screw over it ( - Fig. 4). The final bone crest width achieved was 2 to $3 \mathrm{~mm}$ greater than the initial crestal bone available. The space between the implant and the

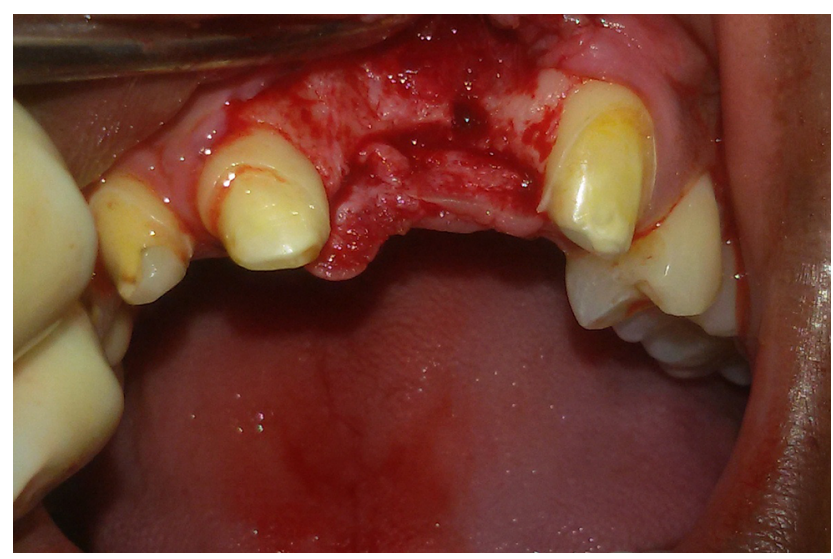

Fig. 2 Midcrestal incision given raising a full thickness mucoperiosteal flap. 


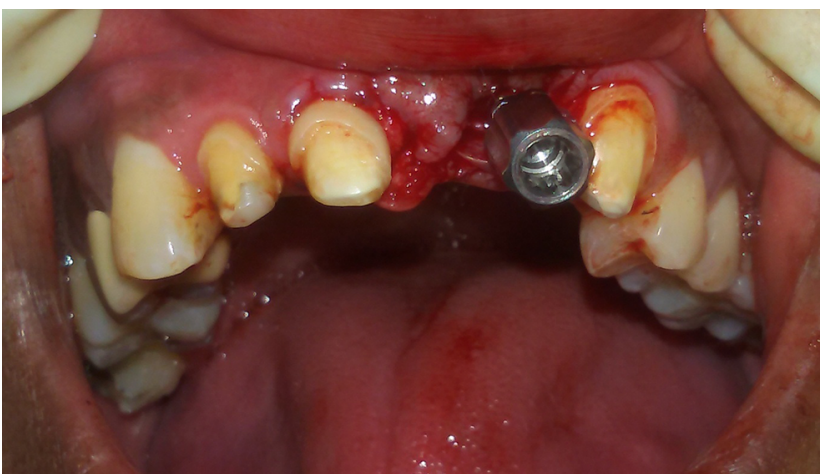

Fig. 3 Bone expander (bone compression kit, MIS Implant, Israel) was inserted at particular intervals.

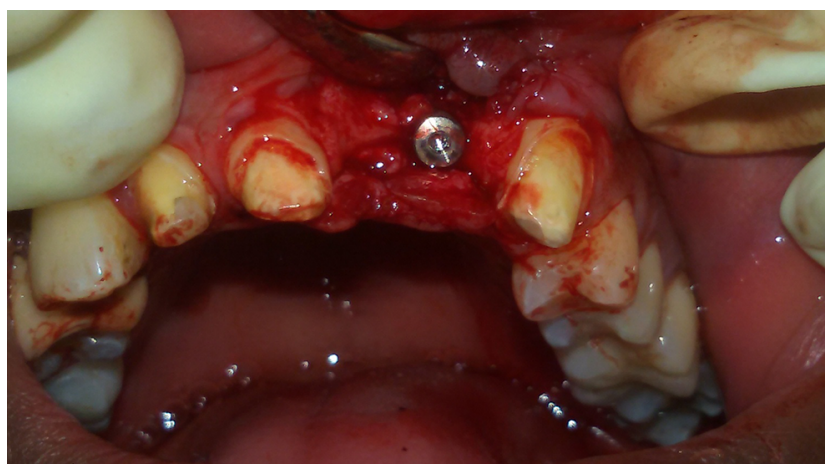

Fig. 4 Single implant with healing abutment (Alpha Biocare SP, Israel) placed in edentulous area.

labial cortical plate was filled with an osseous graft material, that is, allograft (Ostin, Basic OsteoINtegration, Chandigarh, India) and stablilized with a resorbable collagen membrane (Cologide GTR Membrane, Cologenesis, Tamil Nadu, India) (-Fig. 5). Flap was extended coronally over the implant by using periosteal releasing incision so that free interrupted suturing for close approximation can be done ( - Fig. 6 ). Four months post implant placement, osseointegration was evaluated clinically as well as radiographically and implant was found rigidly fixed with an adequate zone of healthy, keratinized gingival without any sign of crestal bone loss and the implant was ready to receive the prosthesis ( - Fig. 7 ).

\section{Restorative Procedure}

Postoperatively, soft tissues in the operated region were analyzed. Gingival margin between the right central incisor and lateral incisor was found to be slight asymmetrical, whereas gingival zenith coincided with the long axis of tooth which made it look nonaesthetic. So, crown lengthening was done, followed by all ceramic crowns for restoring the aesthetics. Teeth preparations were refined in respect to 11,12, and 23 . Provisional restoration was fabricated and was shown to the patient for his acceptance. Using this provisional restoration, a silicon putty index was made to aid in the fabrication of final restoration. Impression transfer was attached to the implant and a final impression was made using polyvinyl siloxane impression material (3M ESPE Soft Putty, 3M India) in a custom tray (-Fig. 8). Two weeks later, the gingival former

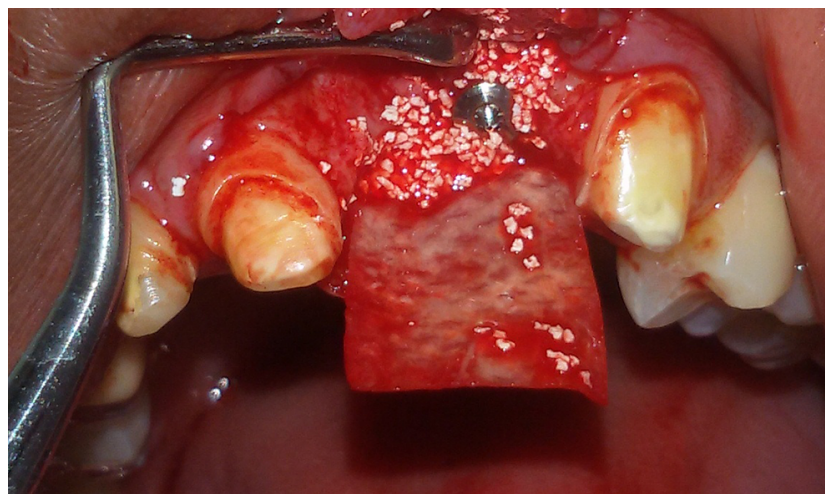

Fig. 5 Allograft (Ostin, Basic OsteolNtegration, Chandigarh, India) placed and stablilized with a resorbable collagen membrane (Cologide GTR Membrane, Cologenesis, Tamil Nadu, India).

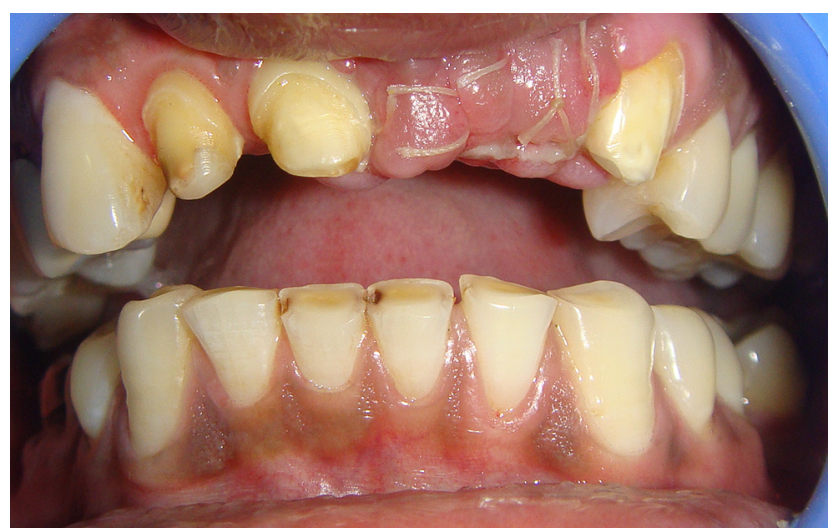

Fig. 6 Interrupted suturing done for close approximation of soft tissue.

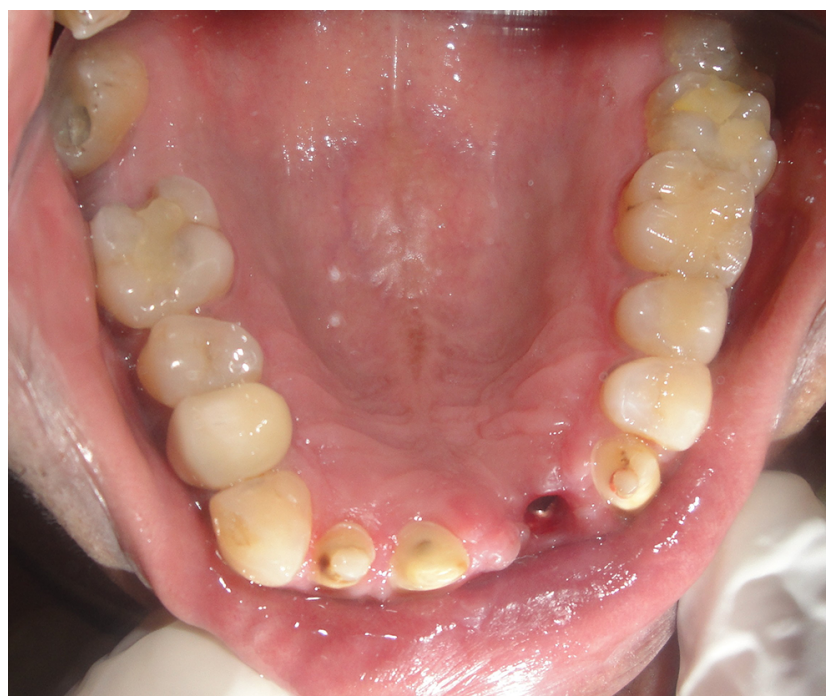

Fig. 7 Intraoral view showing implant surrounded by healthy keratinized gingiva (gingival cuff) after 4 months.

was removed and the soft tissue was analyzed. Definitive abutment was tried onto the implant (-Fig. 9). Ceramic copings were fabricated using computer-aided designing/ computer-aided manufacturing (CAD/CAM) and were then tried in for accurate fit. The copings were sent back to the laboratory for final layering ( - Fig. 10A, B). All ceramic 


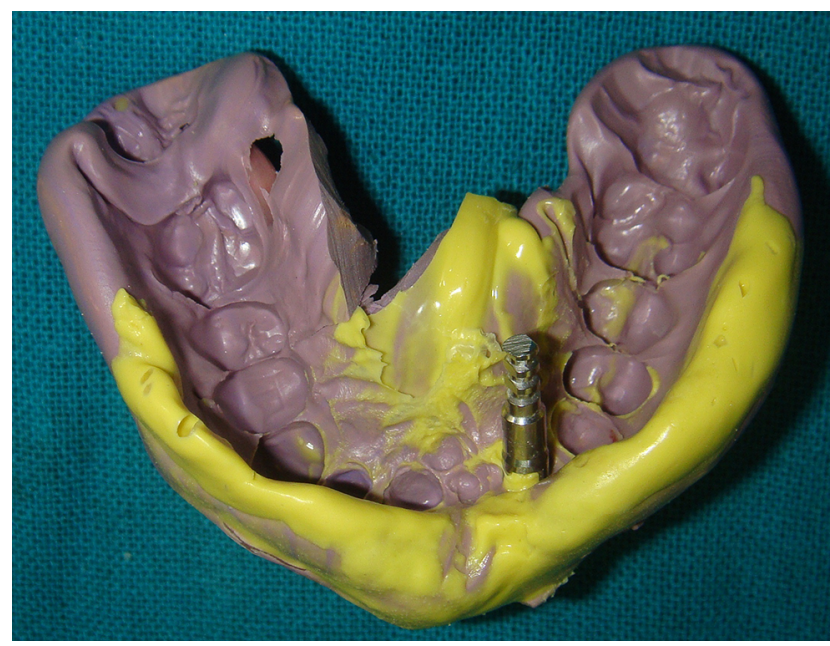

Fig. 8 Final impression made with PVS impression material (3M ESPE Soft Putty, 3M India).
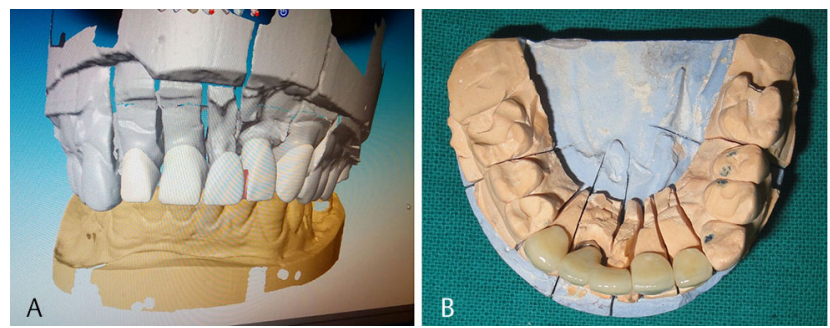

Fig. 10 (A) CAD/CAM used for fabrication of ceramic copings. (B) Finished ceramic crowns received and ready for cementation.

crowns were received and were cemented ( - Fig. 11A, B). The fit of the prosthesis was verified radiographically. Fixation screws were then tightened on the implants to 30 $\mathrm{Ncm}$ using a torque wrench following the cementation of ceramic with a resin cement, thereby checking and adjusting occlusion wherever needed. The patient was recalled for follow-up and was satisfied with the enhanced aesthetics.

\section{Discussion}

Aesthetics is well known to be the art and nature of making a thing look "beautiful." In dentistry, any prosthetic restoration which is properly blended with natural dentition contributes

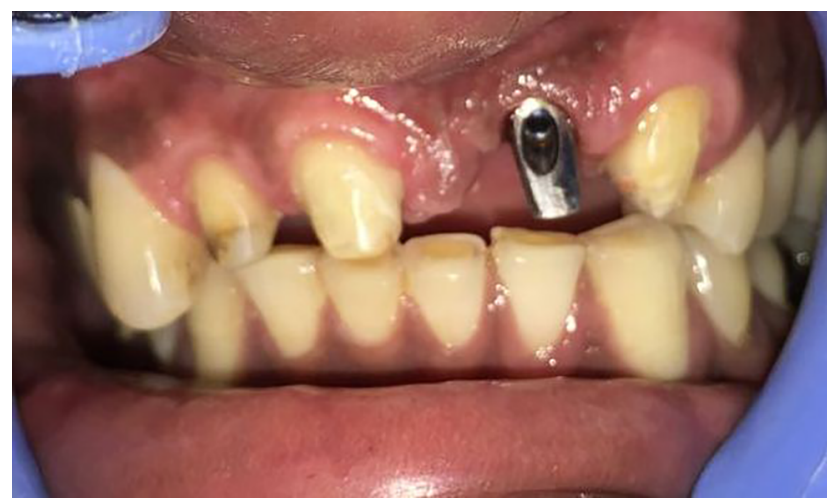

Fig. 9 Definitive abutment was tried onto the implant.

to better aesthetic form and function to the masticatory system. ${ }^{6}$

Certain parameters like gingival and tooth morphology, their texture, dimensions of a clinical crown available, and so forth control the aesthetic value of a fixed restoration. These parameters must be kept in mind giving gingival aesthetics equal importance while restoring any anterior tooth so that any unattractive soft tissue does not result in an unaesthetic result. $^{7}$

A patient's treatment plan should not only be focused on an individual tooth to be restored but rather the entire face should be evaluated. Treatment must be performed in an orderly fashion with proper analysis, that is, by evaluating the facial, dentolabial, phonetic, dental, and gingival parameters. ${ }^{6}$ Nowadays, replacement of missing teeth by an implant is a more acceptable treatment modality. However, the availability of adequate bone volume for dental implant placement in aesthetic zone is often diminished by tooth loss associated with trauma in many young healthy individuals. Loss of buccopalatal dimension of ridge further necessitates calls for additional procedure to receive optimum implant-borne prosthesis. Managing such bony defects in an aesthetic zone becomes more critical as additional bone augmentation procedures are often needed to reconstruct the defect. Various ridge augmentation procedures are utilized to restore bone volume. Guided bone regeneration (GBR), interpositional inlay grafting, onlay grafting, and distraction osteogenesis are some of these procedures. But all of these procedures are relatively aggressive and technique sensitive. ${ }^{8}$
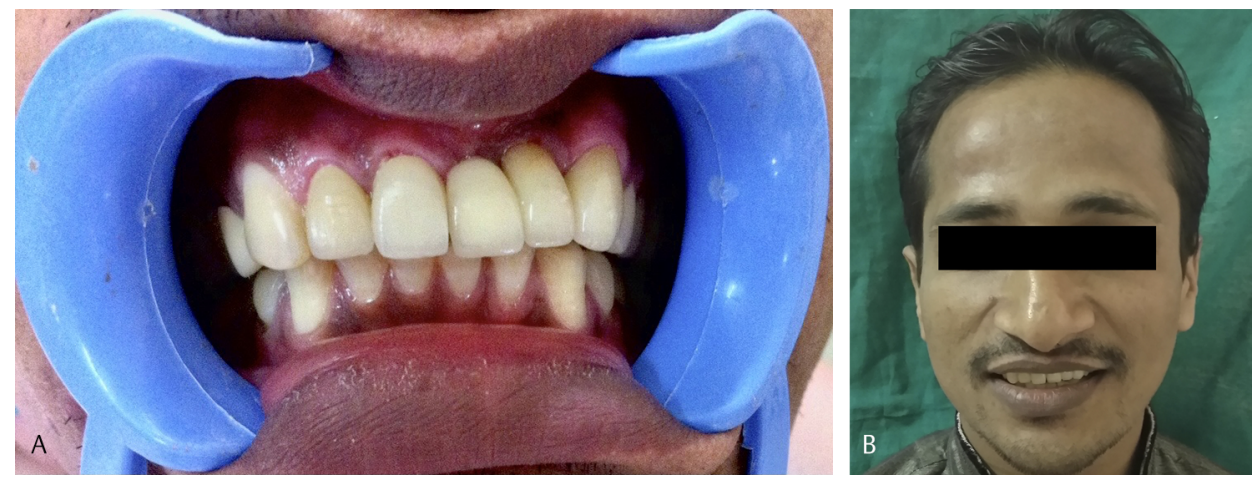

Fig. 11 (A) Final prosthesis placed-intraoral view. (B) Postoperative-extraoral view. 
The alveolar ridge splitting/expansion technique is proposed to create "self-space-making defects" within the bony atrophic rests. The buccal cortical plate is repositioned laterally with a greenstick fracture to create a new implant bed by longitudinal osteotomy of the alveolar bone. ${ }^{9}$ The ridge expansion technique has an advantage over other bone manipulation techniques that it has an overall reduced treatment period with less surgical trauma. Moreover, this technique prevents the waiting period for bone consolidation prior to implant placement with no need of bone harvesting reducing morbidity. In addition, this technique allows a gradual and controlled fracture of the buccal plate if the plastic capacity of the bone has been exceeded. The displacement of the fractured segments may be closely monitored and as long as implant stability is achieved, the fracture site can also be grafted. ${ }^{10}$

\section{Conclusion}

The main challenge in implant-supported restorations for anterior maxilla remains to get a satisfactory aesthetic outcome by appropriate treatment planning and its execution. Factors like tooth and root position of the adjacent teeth, biotype of the periodontium, tooth morphology, smile line, implant site anatomy, implant positioning, and so forth, must be closely monitored for aesthetically pleasing results. With the use of new software-based digital technologies assessment of clinical and biological indices can be accurately performed. Moreover, software can help in virtual planning of implant positioning and implant design for future prosthetic restorations. Osseointegration of dental implants may not be the only focus of a dental practitioner, that is, by improving implant design, surface characteristics, and material bioactivity, but also achieving harmony of an implant restoration with peri-implant soft tissues and bone. Aesthetic parameters such as occlusal space management, occlusal stability, anatomical limits, and periodontal status must be considered prior to plan for any future prosthetic restoration. Further studies are essential to understand the necessary points to be remembered for overall success of an implant placed in the aesthetic zone.

\section{Source of Funding}

None.

\section{Conflict of Interest}

None declared.

\section{References}

1 Forna N, Agop-Forna D. Esthetic aspects in implant-prosthetic rehabilitation. Med Pharm Rep 2019;92(3(Suppl No 3):S6-S13

2 Forna N, Tratat de ProteticăDentară (Treatise of Dental Prosthetics) București: EdituraEnciclopedică; 2011:2

3 Jivraj S, Reshad M, Esthetic implant dentistry: diagnosis and treatment planning. In: Frost RJ, ed. Oral and Maxillofacial Surgery . Netherlands: Elsevier; 2018:391-409

4 Obiechina N. Treatment planning of dental implants in the anterior maxilla; risk assessment and review of soft tissue along with bone preservation and augmentation techniques for successful clinical outcomes. Oral Health and Dental Management 2019;18(2):1-10

5 Hämmerle CHF, Tarnow D. The etiology of hard- and soft-tissue deficiencies at dental implants: a narrative review. J Clin Periodontol 2018;45(Suppl 20):S267-S277

6 Bal A, Dugal R, Shah K, Mudaliar U. Principles of esthetic evaluation for anterior teeth. IOSR J Dent Med Sci 2016;15(3):28-38

7 Hadyaoui D, Dauahi N, Nouira Z, Cherif M. Gingival harmony in anterior aesthetic restorations. Dent J 2014;2(4):155-162

8 McAllister BS, Haghighat K. Bone augmentation techniques. J Periodontol 2007;78(3):377-396

9 Piccinini M. Mandibular bone expansion technique in conjunction with root form implants: a case report. J Oral Maxillofac Surg 2009;67(9):1931-1936

10 Pippi R. Post-surgical clinical monitoring of soft tissue wound healing in periodontal and implant surgery. Int J Med Sci 2017;14(8):721-728 